H. Umemura

Nagoya Math. J.

Vol. 52 (1973), 47-52

\title{
COHOMOLOGICAL DIMENSION OF GROUP SCHEMES
}

\author{
HIROSHI UMEMURA
}

In Umemura [9], we calculated the invariants algcd $(G), p(G), q(G)$ for a commutative algebraic group $G$. We remark that all the results hold for a group scheme which is not necessarily commutative.

To determine $p(G)$, I cannot succeed in dropping the hypothesis "quasi-projective" but this assumption is satisfied in the characteristic 0 case.

\section{Notation and definition}

(1.1) All schemes are connected and of finite type over a fixed field $k$ which we assume to be algebraically closed. Let $X$ be a scheme. The algebraic cohomological dimension of $X$ denoted by alged $(X)$ is, by definition, $\min \left\{n \in N \mid H^{j}(X, F)=0\right.$ for all $j>n$ and all coherent sheaves $F$ on $X$ \}. We need two more invariants $p(X)$ and $q(X)$ defined by the following equations:

$$
\begin{array}{r}
p(X)=\max \left\{n \in N \cup\{\infty\} \mid H^{i}(X, F) \text { is a finite dimensional } k\right. \text {-vector space } \\
\text { for all } i<n \text { and all locally free sheaves } F \text { on } X\} . \\
q(X)=\min \left\{n \in N \cup\{-1\} \mid H^{i}(X, F) \text { is a finite dimensional } k\right. \text {-vector } \\
\text { space for all } i>n \text { and for all coherent sheaves } F \text { on } X\} .
\end{array}
$$

Let $Y$ be a complex analytic space then the analytic cohomological dimension of $Y$ denoted by ancd $(Y)$ is by definition $\min \left\{n \in N \mid H^{i}(Y, F)\right.$ $=0$ for all $i>n$ and all coherent sheaves $F$ on $Y$ \}.

(1.2) Remark 1. Since a quasi-coherent sheaf is a direct limit of coherent sheaves and the functor $H^{i}(X, \quad)$ commutes with direct limits, $\operatorname{algcd}(X)=\min \left\{n \in N \mid H^{i}(X, F)=0\right.$ for all $i>n$ and all quasi-coherent sheaves $F$ on $X\}$.

Remark 2. Let $F$ be a coherent sheaf on $X$, then $F$ has a filtration

Received February 26, 1973. 
such that each of the quotients is a coherent sheaf on $X_{\text {red }}$. Conversely a coherent sheaf on $X_{\text {red }}$ is naturally a coherent sheaf on $X$. Hence $\operatorname{algcd}(X)=\operatorname{algcd}\left(X_{\text {red }}\right)$ and $q(X)=q\left(X_{\text {red }}\right)$.

\section{Algebraic cohomological dimension}

(2.1) THEOREM 1. Let $G$ be a group scheme. Then we have:

$\operatorname{algcd}(G)=\max \{\operatorname{dim} A \mid A$ is an abelian variety such that there exists a surjective homomorphism of group schemes $G_{\text {red }}$ $\rightarrow A\}$

$$
\begin{aligned}
& p(G)= \begin{cases}0 & \text { if } G \text { is quasi-projective and not complete } \\
\infty & \text { if } G \text { is complete }\end{cases} \\
& q(G)= \begin{cases}\operatorname{algcd}(G) & \text { if } G \text { is not complete } \\
-1 & \text { if } G \text { is complete. }\end{cases}
\end{aligned}
$$

Proof. We proved this theorem for commutative algebraic groups in Umemura [9]. In view of Remark 2, to prove the assertions concerning alged $(G)$ and $q(G)$, we may assume that $G$ is reduced. If $G$ is complete, $H^{i}(G, F)$ is finite dimensional for all $i$ and all coherent sheaves and by Lichtenbaum's theorem (Hartshorne [4]) we have algcd $(G)=$ $\operatorname{dim} G$ and $q(G)=-1$. We may also assume $G$ is not complete. First we prove the assertions on $\operatorname{algcd}(G)$ and $q(G)$ under the hypothesis that $G$ is reduced and not complete. Then by Chevalley's theorem we have an exact sequence

$$
1 \longrightarrow B \longrightarrow G \stackrel{\pi}{\longrightarrow} A \longrightarrow 1 \text {. }
$$

where $B$ is an affine group scheme and $A$ is an abelian variety. Since the morphism $\pi$ is affine, we have $H^{i}(G, F)=H^{i}\left(A, \pi_{*} F\right)$ for a coherent sheaf $F$ on $G$. Since $\pi_{*} F$ is quasi-coherent, we have $\operatorname{algcd}(G) \leq \operatorname{dim} A$. In general we have $q(G) \leq \operatorname{algcd}(G)$ from the definition. It is sufficient to show that $q(G)=\operatorname{algcd}(G)=\operatorname{dim} A$. Let $n$ be the dimension of $A$. We have to prove that there exists a coherent sheaf $F$ on $G$ such that $H^{n}(G, F)$ is an infinite dimensional $k$-vector space. We need

THEOREM (Rosenlicht [8] p. 432). Let $C$ be the center of $G$. Then $G / C$ is a linear algebraic group.

COROLLARY. The restriction of $\pi$ to $C$ is surjective. 
Proof of the corollary. By the above Theorem $G / C$ is linear. $A / \pi(C)$ is an abelian variety. Hence the surjective homomorphism $G / C \rightarrow A / \pi(C)$ is trivial and we have $A=\pi(C)$.

If $C$ is not complete, by Umemura [9] 2.7 Corollaire 1, there exists a coherent sheaf $F$ on $C$ and an integer $m \geq n$ such that $H^{m}(C, F)$ is infinite dimensional. $F$ can be regarded as a coherent sheaf on $G$ and we have $H^{m}(G, F)=H^{m}(C, F)$. As we have seen above alged $(G) \leq n$. We conclude that $m=n$. Hence the coherent sheaf $F$ on $G$ has the required property.

If $C$ is complete, then by Rosenlicht's theorem above, $G / C$ is a linear algebraic group of positive dimension since we assume $G$ is not complete.

$$
1 \longrightarrow C \longrightarrow G \stackrel{\varphi}{\longrightarrow} G / C \longrightarrow 1 .
$$

Since $\varphi$ is flat, by base change theorem, $R^{q} \varphi_{*} O_{G}$ is a locally free sheaf on $G / C$ of rank $\left(\begin{array}{c}\operatorname{dim} C \\ q\end{array}\right)$ (see Mumford [6] p. 50 Corollary 2 and p. 129 Corollary 2). Since $G / C$ is affine, we have $H^{0}\left(G / C, R^{q} \varphi_{*} O_{G}\right) \simeq H^{q}\left(G, O_{G}\right)$ by E. G. A. III (1.4.11). Let $m$ be the dimension of $C$. Then $R^{m} \varphi_{*} O_{G}$ is locally free sheaf of rank 1 and $H^{\circ}\left(G / C, R^{m} \varphi_{*} O_{G}\right)$ is infinite dimensional since $G / C$ is affine and of positive dimension. Hence $H^{m}\left(G, O_{G}\right)$ is an infinite dimensional $k$-vector space. It is sufficient to show that $m=$ $\operatorname{dim} C=\operatorname{dim} A$. In fact the restriction of $\pi$ to $C$ is an isogeny of abelian varieties $C$ and $A$. The restriction of $\pi$ to $C$ is surjective by the Corollary above and its kernel $C \cap B$ is finite.

Now we calculate $p(G)$. If $G$ is complete, the assertion is well known. So we may assume $G$ is not complete but quasi-projective. Since $G_{\text {red }}$ is not complete, $G_{\text {red }}$ contains an affine closed subgroup $H$ of positive dimension by Chevalley's theorem. Let $L$ be an ample line bundle on $G$. We denote by $I$ the ideal sheaf of $H$ in $G$. So we have an exact sequence:

$$
0 \longrightarrow I \longrightarrow O_{G} \longrightarrow O_{H} \longrightarrow 0 \text {. }
$$

We have $H^{1}\left(G, I \otimes L^{\otimes \ell}\right)=0$ for a sufficiently large integer $\ell$ since $L$ is ample. We fix such an integer $\ell$. Tensoring $L^{\otimes \ell}$ with $(c)$, we have

$$
0 \longrightarrow I \otimes L^{\otimes \ell} \longrightarrow L^{\otimes \ell} \longrightarrow O_{H} \otimes L^{\otimes \ell} \longrightarrow 0 \text {. }
$$

The exact sequence of cohomology is

$$
H^{0}\left(L^{\otimes \ell}\right) \longrightarrow H^{0}\left(O_{H} \otimes L^{\otimes \ell}\right) \longrightarrow H^{1}\left(I \otimes L^{\ell}\right)=0 .
$$


Since $H$ is affine and of positive dimension and since $O_{H} \otimes L^{\otimes \ell}$ is a line bundle, $H^{0}\left(O_{H} \otimes L^{\otimes \ell}\right)$ is infinite dimensional. By the exact sequence (d), $H^{0}\left(G, L^{\otimes \ell}\right)$ is infinite dimensional. Hence $p(G)=0$. This completes the proof of the Theorem.

(2.2) Remark. I don't know if every group scheme over an algebraically closed field $k$ is quasi-projective. If $G$ is reduced, then $G$ is quasi-projective( Chow [2]). If the characteristic of $k$ is 0 , a group scheme is reduced (Oort [7]). Hence a group scheme is quasi-projective in characteristic 0 .

\section{Analytic cohomological dimension}

(3.1) We need Matsushima's results (Matsushima [5]).

THEOREM A. Let $G$ be a complex Lie group and $N$ a normal subgroup of $G$. We suppose the quotient group $G / N$ is a complex torus $T$. Let $\varphi: N \rightarrow G L(m, C)$ be a linear representation of $N$. Then the principal $G L(m, C)$-bundle on $T$ associated to this representation has a holomorphic connection.

THEOREM B. An indecomposable principal $G L(m, C)$-bundle $P$ over a complex torus with a holomorphic connection can be written in the form;

$$
P=P_{1} \otimes P_{2}
$$

where the transition matrices of $P_{1}$ are upper triangular matrices whose diagonal components are 1 and $P_{2}$ is a principal $C^{*}$-bundle with trivial Chern class.

Corollary. A principal $G L(m, C)$-bundle over a complex torus $T$ with a holomorphic connection is $C^{\infty}$-trivial.

Proof of Corollary. We may assume that $P$ is indecomposable. Then $P$ is isomorphic to $P_{1} \otimes P_{2}$ by Theorem $\mathrm{B}$. It is easy to see that $P_{1}$ and $P_{2}$ are $C^{\infty}$-trivial.

(3.2) THEOREM 2. Let $G$ be a group scheme defined over $C$. Then $\operatorname{algcd}(G) \geq \operatorname{ancd}\left(G^{a n}\right)$.

Proof. By (2.2) $G$ is reduced. By Chevalley's theorem, we have an exact sequence (a). $B$ is a closed sub-group scheme of $G L(m, C)$ for a certain number $m$. Hence we can associate to this representation the 
principal $G L(m, C)$-bundle $\boldsymbol{P}_{G}$ over $A$. By Theorem A, $P_{G}$ has a holomorphic connection. Hence by the Corollary to Theorem B. $P_{G}$ is $C^{\infty}$-trivial. On $A \times G L(m, C)$, we put

$$
f\left(z, x_{11}, \cdots, x_{i j}, \cdots, x_{m m}\right)=\sum_{1 \leq i, j \leqslant n}\left|x_{i j}\right|^{2}+\mid \operatorname{det}\left[\left.\begin{array}{ccc}
x_{11} & \cdots & x_{1 m} \\
\vdots & & \vdots \\
x_{m 1} & \cdots & x_{m m}
\end{array}\right|^{2}\right.
$$

where

$$
\left(z,\left[\begin{array}{ccc}
x_{11} & \cdots & x_{1 m} \\
\vdots & & \vdots \\
x_{m 1} & \cdots & x_{m m}
\end{array}\right]\right) \in A \times G L(m, C) .
$$

Let $g$ be a $C^{\infty}$-isomorphism from the principal $G L(m, C)$-bundle $P_{G}$ to $A \times G L(m, C)$. Let $F$ be the composition $f \circ g$. Then it is easy to see that the closed analytic sub-set $G^{a n}$ of $P_{G}$ is $\operatorname{dim} A+1$-complete by considering the restriction of $f \circ g$ to $G^{a n}$ (cf. Umemura [9]). Hence by a theorem of Andreotti and Grauert [1] p. 250, we have ancd $\left(G^{a n}\right) \leq \operatorname{dim} A$. On the other hand $\operatorname{alged} G=\operatorname{dim} A$ by Theorem $1 . \quad$ q.e.d.

(3.3) Application. Hartshorne's conjecture is true for group schemes. (cf. Hartshorne [4], p. 230 and Umemura [9]).

CoRollary to THEOREM 1 AND THEOREM 2 (Hartshorne's conjecture). Let $G$ be a group scheme over $C$. Consider the natural maps

$$
\alpha_{i}: H^{i}(G, F) \longrightarrow H^{i}\left(G^{a n}, F^{a n}\right)
$$

for any coherent sheaf $F$ on $G$.

(1) $\alpha_{i}$ is an isomorphism for all $i<p(G)$.

(2) $\alpha_{i}$ is an isomorphism for all $i>q(G)$.

(3) $F \mapsto F^{a n}$ is an equivalence of the category of coherent algebraic sheaves on $G$ and the category of coherent analytic sheaves on $G^{a n}$ if $p(G) \geq 1$.

Proof. If $G$ is complete, we have nothing to prove. If $G$ is not complete, $p(G)=0$ by Theorem 1 . Hence (1) and (3) are trivial. $q(G)$ $=\operatorname{algcd}(G)$ by Theorem 1, and $\operatorname{algcd}(G) \geq \operatorname{ancd}\left(G^{a n}\right)$ by Theorem 2 . Hence (2) follows.

(3.4) Remark. In [9], we show that, for any integer $n \geq 0$, there exists an algebraic variety (indeed, a commutative algebraic group) $G$ 
defined over $C$ such that alged $(G)=n$ and ancd $\left(G^{a n}\right)=0$. By considering the product with a complete variety, for any pair of integers $n \geq$ $m \geq 0$, there exists an algebraic variety $G$ such that $\operatorname{algcd}(G)=n$ and $\operatorname{ancd}\left(G^{a n}\right)=m$.

\section{REFERENCES}

[1] Andreotti, A. and Grauert, H.: Théorème de finitude pour la cohomologie des espaces complexes, Bull. Soc. Math. France, 90 (1962), 193-259.

[2] Chow, W. L.: On the projective embedding of homogeneous varieties. A symp. in honor of S. Lefschetz, 122-128, Princeton University press.

[ 3 ] Dieudonné, J. et Grothendieck, A.: E.G.A.

[4] Hartshorne, R.: Ample subvarieties of algebraic varieties, Lecture notes in mathematics 156, Springer.

[5] Matsushima, Y.: Fibrés holomorphes sur un tore complexe, Nagoya Math. J., 14, 1-24 (1959).

[6] Mumford, D.: Abelian varieties, Oxford University press.

[ 7 ] Oort, F.: Algebraic group schemes in characteristic zero are reduced, Inventiones math., 2 (1966), 79-80.

[ 8 ] Rosenlicht, M.: Some basic theorems on algebraic groups, Amer. J. of Math., 78 (1956), 401-443.

[9] Umemura, H.: Dimension cohomologique des groupes algébriques commutatifs, Ann, Scien. de l'Ecole Normale Supérieure, $4^{\mathrm{e}}$ series 5 (1972), 265-276.

[10] — : La dimension cohomologique des surfaces algébriques, Nagoya Math. J., 47 (1972), 155-160.

Department of Mathematics

Nagoya University 